I. Gen. Appl. Microbiol.

Vol. 5, Nos. 1 2, 1959

\title{
PREPARATION OF L-ASPARTIC ACID BY BACTERIAL ASPARTASE (Preliminary Report)
}

\author{
KAKUO KITAHARA, SAKUZO FUKUI and MASANARU MISAWA \\ Division of Zymomycology, the Institute of Applied Microbiology, \\ University of Tokyo \\ Received for publication, March 20, 1959
}

Since the discovery of aspartase in Escherichia coli by J. H. QuASTEL and B. Woolf in 1926 (1), bacterial aspartase has been investigated by many workers (2-5). Most work has been carried out from the stand point of nitrogen metabolism exclusively, and it is hard to find any report dealing with attempts to apply this enzyme to the commercial production of $\mathrm{L}$-aspartic acid.

Recently, the production of glutamic acid and some other amino acids has become available by fermenting process. The present authors have searched for an enzymic process applicable for L-aspartic acid synthesis in industrial manufacture. In this paper, the selection of a suitable microorganism and its reaction conditions are presented.

\section{Selection of Strains}

For the purpose of obtaining suitable strains the following method was employed. Each strain tested was incubated in a malt-bouillon medium ${ }^{1}$ adjusted near its optimum temperature. After 24 hours the substrate solution containing same amount of $\mathrm{Na}$-fumarate and ammonium chloride was added to the culture broth so as to make the final concentration of fumaric acid to $2 \%$, and incubation was continued further for 1 day at $37^{\circ}$, the optimum temperature for aspartase reaction.

Fumaric acid in the reaction mixture was determined by permanganometry (6) and aspartic acid was roughly estimated by paperchromatography.

As summarized in Table 1, it is found that Escherichia coli No. 2, Serratia marcescens and Bacterium succinicum apper to be strains suitable for our purpose.

Enzymic Synthesis of $L$-Aspartic Acid

Enzyme preparation: Cells of Escherichia coli No. 2, propagated in a

1. Malt-bouillon medium: an equivolume mixture of malt extract and nutrient bouillon. 
Table 1. Selection of Aspartase Forming Strain.

\begin{tabular}{|c|c|c|}
\hline Organism & $\begin{array}{l}\text { Fumaric acid } \\
\text { consumed. } \%\end{array}$ & $\begin{array}{c}\text { Spot of } \\
\text { aspartic acid* }\end{array}$ \\
\hline Pseudomonas ovalis & 87.4 & +++ \\
\hline Pseudomonas xanthe & 56.1 & \\
\hline Achromobacter cycloclaster & 53.3 & \\
\hline Alcaligenes faecalis & 3.8 & \\
\hline Flavobacterium lactis & 42.3 & \\
\hline Serratia marcescens & 90.2 & $++t+$ \\
\hline Erwinia carotovora & 23.6 & ++ \\
\hline Aerobacter aerogenes & 49.5 & +++ \\
\hline Aeromonas hydrophila & 91.3 & +++ \\
\hline Escherichia coli No. 2 & 91.7 & $++t+$ \\
\hline Escherichia coli $\mathrm{K}_{12}$ & 1.1 & \\
\hline Agrobacter tumefaciens & 37.4 & + \\
\hline Aerobacter cloacae & 8.3 & + \\
\hline Micrococcus lysodeikticus & 9.3 & \\
\hline Micrococcus luteus & 4.9 & \\
\hline Lactobacillus casei & 0 & \\
\hline Propionibacterium arabinosum & 59.9 & \\
\hline Bacillus subtilis & 75.3 & \\
\hline Bacillus coagulans & 0 & \\
\hline Bacterium succinicum & 90.1 & $++t+$ \\
\hline Streptomyces griseus & 7.7 & \\
\hline Nocardia asteroides & 7.7 & + \\
\hline Beer yeast 1 & 11.5 & + \\
\hline Beer yeast 2 & 25.3 & \\
\hline Wine yeast & 15.9 & \\
\hline Willia saturnus & 67.2 & \\
\hline
\end{tabular}

* Paper chromatography is carried on as follows: Tōyō filter paper No. 50; solvent system, n-butyl alcohol-acetic acid-water (4:1:1).

medium for 2 days at $37^{\circ}$, were harvested by centrifugation, washed once with distilled water and lyophilized. The yield of the dried cells was approximately $0.7 \mathrm{~g}$ per 1 liter culture broth. 'This dried preparation was used as the enzyme material throughout this work.

Composition of reaction media:

Medium 1, Sodium-fumarate

Ammonium chloride

$10 \mathrm{~g}$ (free acid basis)

Dried cells (enzyme material) $0.25 \mathrm{~g}$

Distilled water $\quad 100 \mathrm{ml}$

Medium 2, Ammonium-fumarate $20 \mathrm{~g}$ (free acid basis)

Dried cells $0.5 \mathrm{~g}$

Distilled water $\quad 100 \mathrm{ml}$ 
Medium 3, Ammonium-fumarate Dried cells

Distilled water

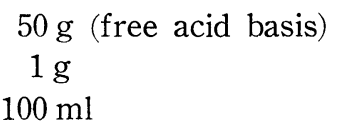

$50 \mathrm{~g}$ (free acid basis)

$100 \mathrm{ml}$

Note: The ammonium-fumarate in Medium 3 was prepared as a saturated solution containing an abundant amount of crystals. These crystals were resolved into the solution accompanied with the proceeding of enzyme reaction.

Reaction was carried on with slow agitation at $37^{\circ}$ and $\mathrm{pH}$ 7.2-7.4. After 24 hours, almost all of the fumarate disappeared and was converted into L-aspartic acid. By simple adjustment of the hydrogen ion concentration to the isoelectric point, $\mathrm{pH} 2.77$, the majority of the product was crystallized and could be readily separated from the reaction mixture by filtration. The experimental results are presented in Table 2.

Table 2. Enzymic Synthesis of L-Aspartic Acid.

\begin{tabular}{|c|c|c|c|c|c|c|}
\hline & \multirow{2}{*}{\multicolumn{2}{|c|}{$\frac{\text { Medium } 1}{\text { Na-salt }}$}} & \multicolumn{2}{|c|}{ Medium 2} & \multicolumn{2}{|c|}{ Medium 3} \\
\hline & & & \multicolumn{4}{|c|}{$\mathrm{NH}_{4}$-salt } \\
\hline & $\underset{\mathrm{g}}{\text { Fumaric }}$ & $\begin{array}{c}\text { Aspartic } \\
\mathrm{g}\end{array}$ & $\begin{array}{c}\text { Fumaric } \\
\mathrm{g}\end{array}$ & $\begin{array}{c}\text { Aspartic } \\
\mathrm{g}\end{array}$ & $\underset{\mathrm{g}}{\text { Fumaric }}$ & $\underset{\mathrm{g}}{\text { Aspartic }}$ \\
\hline \multicolumn{7}{|l|}{ Initial } \\
\hline Substrate & 10.0 & & 20.0 & & 50.0 & \\
\hline Loss of determination & 0.21 & & 0.52 & & 0.59 & \\
\hline Difference & 9.79 & & 19.48 & & 49.41 & \\
\hline \multicolumn{7}{|l|}{ Final* } \\
\hline Crystals & 0 & 8.72 & 0 & 19.69 & 0.2 & 51.20 \\
\hline Mother liquid & 0.32 & 1.50 & 0.07 & 1.15 & 0.1 & 2.61 \\
\hline Loss of determination & & 0.31 & & 1.40 & & 2.21 \\
\hline Total aspartic & & 10.53 & & 22.19 & & 56.02 \\
\hline \multicolumn{7}{|l|}{ Yield } \\
\hline $\begin{array}{l}\text { Formed aspartic per } \\
\text { comsumed fumaric } \\
\text { (mol./mol.) }\end{array}$ & \multicolumn{2}{|c|}{$93.9 \%$} & \multicolumn{2}{|c|}{$99.3 \%$} & \multicolumn{2}{|c|}{$99.4 \%$} \\
\hline $\begin{array}{l}\text { Recovered aspartic } \\
\text { as crystals (mol./mol.) }\end{array}$ & \multicolumn{2}{|c|}{$77.8 \%$} & \multicolumn{2}{|c|}{$88.1 \%$} & \multicolumn{2}{|c|}{$90.4 \%$} \\
\hline
\end{tabular}

Determination of L-aspartic acid: The colorimetric method( $(7)$ was employed.

* After the reaction and $\mathrm{pH}$ adjustment

The recrystallized product from water showed a melting point of $270^{\circ}$.

Anal. Calcd. for $\mathrm{C}_{4} \mathrm{H}_{7} \mathrm{NO}_{4}$
C $36.13 \%$ H $5.27 \%$ N $10.46 \%$
Found
C $36.09 \%$ H $5.30 \%$ N $10.52 \%$

Specific rotation, $[\alpha]_{\mathrm{D}}^{1: 0}=+24.0^{\circ}(\mathrm{C}=1$ in $1.91 \% \mathrm{HCl})$

In conclusion the present authors wish to state that, if a suitable enzyme 
preparation and its reaction condition can be selected, some of the enzymes other than hydrolase will also be promising for industrial manufacture. Bacterial aspartase, for example, will be the most advantageous tool for the production of L-aspartic acid because it shows not only full activity in the saturated ammonium-fumarate solution but also shows an equilibrium constant shifting to the direction of aspartic acid formation.

\section{REFERENCES}

(1) J. H. Quastel and B. Woolf: Biochem. J., 20, 545 (1926).

(2) E. F. GALE: Biochem. J., 32, 1583 (1938).

(3) A. I. Virtanen and J. TARnANen: Biochem. Z., 250, 193 (1932).

(4) Y. Sumiki: Bull. Japan. Soc. Ferment., 23, 33 (1928).

(5) Nils Ellfolk: Acta Chemica Scandinavica, 8, 151 (1954).

(6) F. B. Straub: Z. physiol. Chem., 336, 43 (1935).

(7) E. W. Yемm and E. C. Cocking: Analyst, 80, 209 (1955). 